\title{
Theoretical investigation of fiber Raman amplifier with dynamic gain control
}

\author{
Xiang Zhou, Hossam H. M. Shalaby, Chao Lu, Ping Shum and Teehiang Cheng \\ Optical network group, Nanyang Technological Univ., School of Electrical and Electronic Engineering, Block S2, \\ NTRC, Nanyang Avenue, Singapore 639798 \\ Fax:65-7926894,Email: ezhou@ntu.edu.sg
}

\begin{abstract}
A gain-clamped fiber Raman amplifier is proposed and simulated. The results show that, gain variation of less than $0.2 \mathrm{~dB}$ over $70 \mathrm{~nm}$ bandwidth is obtainable for signal power variation of $18 \mathrm{~dB}$. (C) 2001 Optical Society of America

OCIS codes: (060.2320) Fiber optics amplifiers and oscillators
\end{abstract}

\section{Introduction}

Among the various competing candidates for next generation fiber amplifier, Fiber Raman amplifiers (RFAs) are recently attracting many researchers' attention in DWDM system due to their distinctive flexibility in bandwidth designs and growing maturity of high power pump module technologies [1-3]. Despite the simplicity of the architecture of a Raman amplifier, many issues, such as gain flattening, double Rayleigh scattering noise, ASE noise must be considered in the design of the amplifier and the systems that use them. Further more, in the design of optical amplified link for DWDM application in a network scenario, the number and the power level of the input channels may vary randomly in time, therefore it is also essential to stabilize the amplifier gain profile.

In this paper, we investigated the issue of dynamic gain control for fiber Raman amplifier through theoretical simulation. The basic idea is similar to a gain-clamped EDFA. That is, by introducing an all-optical feedback lasing signal sustained by the amplifier itself, we hope that the gain profile of a RFA can be clamped to the desired level. To our knowledge, this is the first time this issue is being dealt with.

\section{Principle}

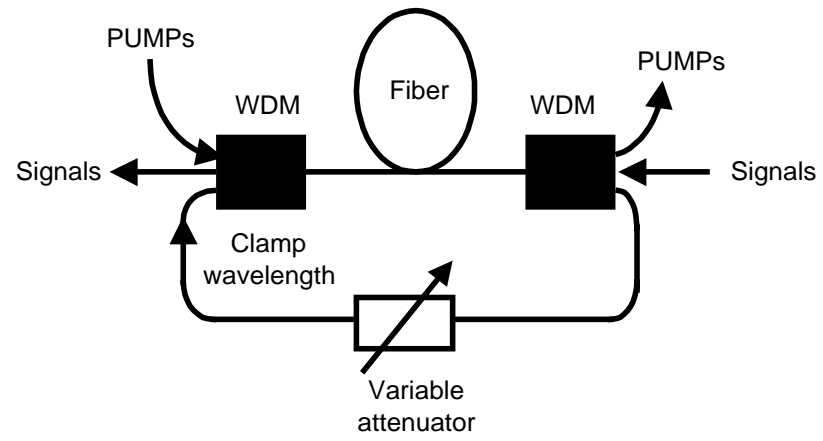

Fig. 1. Diagram of fiber Raman amplifier with automatic gain control

The proposed gain_clamp Raman amplifier is shown in Fig. 1, where both the pumps and the lasing light are designed to counter propagate against the signals (to avoid pump-induced crosstalk). It's clear that the loss of the variable attenuator uniquely determines the gain of the lasing light at a stable state. In order to generate a wide-band and gain-flatten spectrum profile, it is known that, a Raman fiber amplifier usually needs more than two pumps [4]. This is different from the case of EDFA. At a stable state, and neglecting additional various effects such as Rayleigh back scattering, temperature dependent spontaneous Raman emission, and the interaction between signals, the propagation equations governing signal lights and lasing lights power evolution can be simplified in the form as the follows. 


$$
\begin{aligned}
\frac{d S\left(z, v_{i}\right)}{d z}= & \frac{1}{K_{e f f} A_{\text {eff }}}\left(\sum_{j=1}^{P} g_{r}\left(v_{i}-\varsigma_{j}\right) P\left(z, \varsigma_{j}\right)+g_{r}\left(v_{i}-\xi\right) F(z, \xi)\right) S\left(z, v_{i}\right) \\
& \frac{d F(z, \xi)}{d z}=\frac{1}{K_{e f f} A_{e f f}}\left(\sum_{j=1}^{P} g_{r}^{n u m}\left(\xi-\varsigma_{j}\right) P\left(z, \varsigma_{j}\right)\right) F(z, \xi)
\end{aligned}
$$

Here $S\left(z, v_{i}\right), P\left(z, \zeta_{j}\right)$ and $F(z, \xi)$ denote the signal power, pump power and lasing light power, respectively. $g_{r}\left(v_{i}-\zeta_{j}\right), A_{e f f}$ and $K_{\text {eff }}$ represent Raman gain coefficient, effective area of the fiber and polarization-related factor. $P_{-}$num means the number of the used pumps. Integrating (1) and (2) along the fiber length, we get the signal and lasing light gain as

$$
G_{S}\left(v_{i}\right)=\exp \left(\sum_{j=1}^{P} g_{r}\left(v_{i}-\varsigma_{j}\right) H_{P}\left(\varsigma_{j}\right)+g_{r}\left(\xi-\varsigma_{j}\right) H_{F}(\xi)\right)
$$

and

$$
G_{F}(\xi)=\exp \left(\sum_{j=1}^{P} g_{r}\left(\xi-\varsigma_{j}\right) H_{P}\left(\varsigma_{j}\right)\right)
$$

respectively. Here $H_{P}\left(\varsigma_{j}\right)=\int_{0}^{L} P\left(z, \varsigma_{j}\right) d z$ ( $L$ means the fiber length) and $H_{F}(\xi)=\int_{0}^{L} F(z, \xi) d z$. Note that the signal gain is only dependent on the integration of pump power (including lasing light) along the fiber length. Unlike a gain-clamped EDFA, we can see that, even if $G_{F}(\xi)$ remains a constant, the value of $G_{S}\left(v_{i}\right)$ is still uncertain. However, observing (3) and (4) carefully, we can found that, the introduction of lasing light can still reduce the gain fluctuation caused by power variation of the incoming signal light, especially for signals around the wavelength of the lasing light. It can also be supposed that, the "gain-locking" bandwidth will be dependent on the wavelength of the lasing light. To verify these suggestions, we performed computer simulations for both the stable state performance and transient dynamics.

\section{Simulation results and discussions}

We have simulated the performance of the proposed gain_clamped RFA at a stable state. In our simulation, we considered the pump-pump, pump-signal, signal-signal, pump-lasing light, and signal-lasing light interactions, temperature dependent ASE noise and Rayleigh back scattering noise. The parameter used in our simulation is given as follows. Pump wavelength: $1425 \mathrm{~nm}, 1446 \mathrm{~nm}$ and $1480 \mathrm{~nm}$. The corresponding pump power is $0.88 \mathrm{w}, 0.44 \mathrm{w}$, and $0.32 \mathrm{w}$, respectively. 100 incoming signal $\left(1510 \mathrm{~nm}\right.$ to $1610 \mathrm{~nm}, 1 \mathrm{~nm}$ separation). Fiber length $=10 \mathrm{~km}, A_{\text {eff }}=50$ $\mathrm{um}^{2}$ and $K_{\text {eff }}=2$. The Raman gain profile of silicon fiber was chosen from [5]. Rayleigh back scattering coefficient was chosen to be $10^{-7} \mathrm{~km}^{-1}$.

Fig.2 gives the calculated gain spectrum profile and noise figure of a RFA without gain control. We can see that, while incoming signal power varying from $-30 \mathrm{dBm}$ to $-12 \mathrm{dBm}$, the signal gain fluctuation can be more than $8 \mathrm{~dB}$. However, when we introduce a lasing light as shown in Fig. 1, the gain fluctuation can be compressed in a considerable degree. Such a result is clearly shown in Fig. 3, where the lasing wavelength was chosen to be 1605 $\mathrm{nm}$. It can be seen that, gain variation of less than $0.2 \mathrm{~dB}$ can be obtained over $70 \mathrm{~nm}$ bandwidth (from 1540 to 1610). However, when the lasing wavelength is chosen to be shorter, our simulation shows that, the gain-clamping bandwidth will become narrower. For example, for lasing wavelength at $1540 \mathrm{~nm}$, only within 1520 to 1550 , the gain variation can be locked to less than $0.2 \mathrm{~dB}$. Beyond this range, it is found that, signal gain fluctuation is greater at relatively longer wavelength than that at a relatively shorter wavelength.

Similar to the case of a gain-clamped EDFA, from Fig. 3 we can see that, the noise figure will increase definitely when gain compression is large. It is worthnoting that, such a detrimental effect is more serious in the 
region of relatively shorter wavelength. So, there is a trade off between dynamic gain control and noise performance for practical amplifier design.

The transient dynamics of a gain-clamped RFA was also investigated. The detailed results will be presented in the conference.

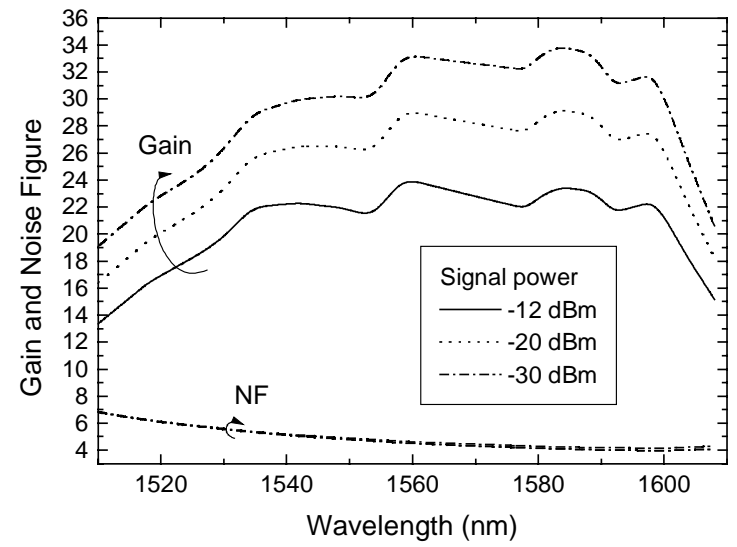

Fig. 2. Gain and noise figure of a conventional Raman fiber amplifier

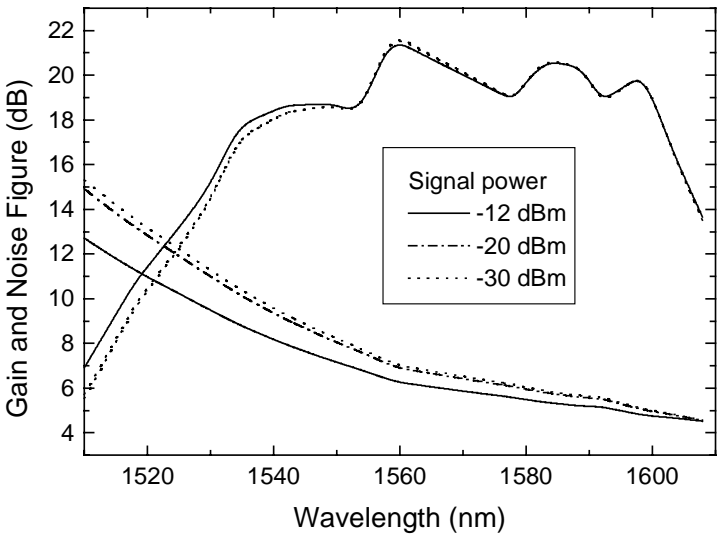

Fig. 3. Gain and noise figure of a gain_clamped Raman fiber amplifier

\section{Reference}

[1] OSA IEEE: F. Koch, S.A.E.Lewis, S.V.Chernikov,J.R.Taylor, "Broadband gain flattened Raman amplifier to extend operation in the third telecommunication window", OFC (Baltimore, USA, 2000), paper FF3-1.

[2] OSA, IEEE : Hiroji Masuda, "Review of wideband hybrid amplifier", ”, OFC (Baltimore, USA, 2000), paper TuA1-1.

[3] P.B.Hansen, L.Eskildsen, S.G.Grubb, A. J.Stentz, T.A.Strasser, J.Judkins, J.J.DeMarco, R.Pedrazzani, and D.J.DiGiovanni , "Capacity upgades of transmission system by Raman amplifier”, IEEE Photonics Technology Lett., Vol. 9, no. 2, pp. 262-264 (1997).

[4] Howard Kidorf, Karsten Rottwitt, Morten Nissov, Matthew Ma and Eric Rabarijaona, "Pump interaction in a 100-nm bandwidth Raman amplifier", IEEE Photonics Technology Letters, Vol. 11, no. 5, pp. 530-532 (1999).

[5] R.H.Stolen, E.P.Ippen, and A.R.Tynes, "Raman oscillation in glass optical waveguide," Appl. Physics Lett., Vol. 20, no. 2 , pp. 62 - 64 (1972). 